http://jmscr.igmpublication.org/home/ ISSN (e)-2347-176x ISSN (p) 2455-0450

crossref DOI: https://dx.doi.org/10.18535/jmscr/v8i6.82

\author{
Journal Of Medical Science And Clinical Research \\ IGM Publication \\ An official Publication of IGM Publication
}

\title{
Research Paper \\ Short Term Neonatal Morbidities of Post-Term Births: A Prospective Cohort Study
}

\author{
Authors \\ Dr Sujit Nath Choudhury, Dr Pranav N Saji
}

\begin{abstract}
Introduction: Post-term pregnancy is defined as pregnancy reaching to or extending beyond 42 weeks of gestation from the last menstrual period. The introduction of routine ultrasound for gestational age dating has significantly reduced the incidence of post-term pregnancy. An increased risk of perinatal mortality and morbidity also found in the post-term babies. Maternal factors also plays a major role in delivery for $\geq 42$ completed gestational weeks.

Materials and Methods: This study is a prospective cohort study conducted on 110 live births born between April 2019 and March 2020 in a tertiary care hospital. Data were collected using a structured questionnaire and twice daily examination of the baby. The complication developed is recorded and detailed maternal history also taken.

Results: The prevalence of post-term births to be 3.9\%. Advanced maternal age, oligohydramnios and previous history of post term delivery were found to be the major factor responsible for the post-term delivery. Meconium staining of amniotic fluid, Meconium aspiration syndrome, birth asphyxia, polycythemia, hypocalcemia and hypoglycemia are found to have higher incidence in post-term babies. Neonatal mortality in post-term neonates in our study was $10.1 \%$.

Conclusion: Primiparity, advanced maternal age and previous post-term delivery were found to be the strongest risk factors for post-term pregnancy. Study also demonstrated an association between post-term pregnancy and an increased risk of adverse neonatal outcome for post-term babies. This should be kept in mind by obstetricians when considering the management of post-term pregnancy as well as by paediatricians who need to be aware of the higher rate of complications in post-term neonates.
\end{abstract}

\section{Introduction}

"Postterm pregnancy" is defined by the World Health Organization as the end of gestation at $\geq 42$ completed weeks' gestation, measured from the first day of the last menstrual period (LMP) and based on a 28 -day cycle ${ }^{1}$. The incidence of postterm pregnancy is about $4-7 \%$ of all pregnancies ${ }^{2}$. The introduction of routine ultrasound for gestational age dating has significantly reduced the incidence of postterm pregnancy ${ }^{3}$.The prevalence of post term pregnancies varies depending on population characteristics and local management practices like percentage of primigravidas, the prevalence of a prior postterm pregnancy as well as genetic predisposition ${ }^{4}$. Several studies have demonstrated an association between post-term pregnancy and an increased risk of adverse neonatal outcome neonates of singleton pregnancies born at $42+0$ weeks' gestation or later had higher rates of neonatal 
convulsions, meconium aspiration syndrome (MAS), higher rates of meconiumstained amniotic fluid (MSAF), neonatal intensive care unit (NICU) admission, and birth aspyxia in post-term neonates versus term neonates ${ }^{5}$. The risk of stillbirth was higher in post-term births than in term births. The incidence of stillbirth increases from 39 weeks onwards with a sharp rise after 40 weeks of gestation ${ }^{6}$. Prompted by these findings, the American College of Obstetricians and Gynecologists (ACOG) recommended that labour be induced after $42+0$ gestational weeks and no later than $42+6$ gestational weeks ${ }^{7}$. The aim of the present study was to describe the outcome of postterm low-risk pregnancy and toexamine the association between post-term delivery and a broad spectrum of neonatal morbidities in a large group of neonates of singleton pregnancies and maternal factors which leads to post-term delivery.

The aim of the present study was to describe the natural history, outcome of post-term babies and to found the association between post-term delivery and a broad spectrum of neonatal morbidities in a large group of neonates of singleton post-term pregnancies.

\section{Materials and Methods}

The present study was a prospective cohort study conducted in a tertiary care hospital in India. The study was carried out for a total duration of one year from April 2019 to March 2020. Last menstrual period is used for dating only when a first trimester ultrasound has not been done. The study group included all singleton neonates born at $\geq 42$ weeks. To isolate the effect of gestational age on pregnancy outcome, we excluded all neonates of pregnancies that met any of the following high-risk criteria: multiple gestation, maternal hypertensive disorders, cholestasis of pregnancy, placental abruption and placenta previa. Every baby admitted in neonatal intensive Care Units are examined twice a day and the complication developed is recorded. All data collected was analysed using SPSS software used for statistical analysis.

\section{Results}

A total of 3662 newborns were admitted during the study period to Neonatal Intensive Care Unit. Out of which 144 are post term babies which constitutes about $3.9 \%$ of total live birth. Among 144 neonates, 110 babies satisfied the inclusion and exclusion criteria. Out of 110 post term babies 59 are males and 51 are females. Mothers in the post-term group had significantly lower parity. Advanced maternal age $>35$ years is found in 54 cases, 40 cases between 25 to 35 and in 16 cases age of the mother is less than 25 years. It was found that 64 mothers are primiparous, 28 are multiparous and 18 are grand multiparous. Most common mode of delivery was normal vaginal delivery $(46.4 \%)$ followed by $45.4 \%$ in caesarian section. $8.2 \% \%$ by assisted modes of delivery [forceps/ instrument assisted vaginal delivery]. Higher rate of induction, instrumental deliveries and Caesarian section are also found in post term group. History of previous post term pregnancies were found in 15 cases (13.6\%). Oligohydramnios is noticed in 28 cases of post-term pregnancies which constitute $25.6 \%$ of study population.

Table: 1 post term delivery in relation with mode of delivery

\begin{tabular}{|l|c|c|}
\hline Mode of delivery & No. of babies & Percent (\%) \\
\hline Vaginal delivery & 51 & $46.4 \%$ \\
\hline Caesarean section & 50 & $45.4 \%$ \\
\hline $\begin{array}{l}\text { Instrumental/Forceps } \\
\text { delivery }\end{array}$ & 9 & $8.2 \%$ \\
\hline
\end{tabular}

It was found that 24 babies are Small for Gestational Age [SGA], 81 are appropriate for gestational age [AGA] and 5 are Large for Gestational Age [LGA].Slight male predilection is seen. 58 were males and 42 were females. Dysmorphicfacies is seen in 3 cases $(2.7 \%)$ and congenital anomaly noticed in 9 cases $(8.1 \%)$. One case of anencephaly also found in post-term age group. 29 babies had a normal post neonatal period while other babies had developed some complication during the study period. Meconium 
staining of amniotic fluid was found in 72 cases, out of which 32 cases developed meconium aspiration syndrome. As a supportive treatment for MAS Oxygen by nasal prongs given for 15 cases, CPAP given for 10 cases and 7 babies were put on mechanical ventilation. Chest X Ray shows normal study (18\%), interstitial infiltrates (50\%), hyperinflation (16\%) and pneumonia (16\%). Higher incidence of birthasphyxia is noticed in post term babies. For meconium aspiration, the lowest rate was found in post-term AGA infants and the highest in post-term AGA infants47 babies developed birth asphyxia of which 19 had no episode of convulsion and only shows some irritability for few hours following resuscitation, 21 neonates comes under stabe 2, 7 neonates classified under stage 3 in Sarnat and Sarnat staging of hypoxic ischemic encephalopathy. The lowest rate of convulsions was found in post-term AGA infants and the highest in post-term SGA infants.

Table: 2 Short term neonatal morbidities of postterm babies

\begin{tabular}{|l|c|c|}
\hline COMPLICATION & No. of babies & Percentage(\%) \\
\hline Birth asphyxia & 47 & $42.7 \%$ \\
\hline $\begin{array}{l}\text { Meconium aspiration } \\
\text { syndrome }\end{array}$ & 32 & $29 \%$ \\
\hline $\begin{array}{l}\text { Persistant pulmonary } \\
\text { hypertension }\end{array}$ & 19 & $17.2 \%$ \\
\hline Hypoglycemia & 11 & $10 \%$ \\
\hline Hypocalcemia & 10 & $9 \%$ \\
\hline Polycythemia & 9 & $8.1 \%$ \\
\hline Congenital anomaly & 9 & $8.1 \%$ \\
\hline
\end{tabular}

During the study period of a year, we enrolled 19(13 males and 6 females) infants with the diagnosis of persistent pulmonary hypertension of newborn. MAS [9 cases] and Birth asphyxia [7 cases] were relatively more associated with PPHN. Metabolic complications like hypocalcemia and hypoglycemia increasingly noticed in the study group. Both the complication are noticed mainly for small for gestational age group babies. Hypoglycemic convulsion occurred in 2 out of 11 cases of post-term babies who developed hypoglycemia. 8 out of the 11 cases were small for gestational age babies.
Hypocalcemia mainly seen for babies who developes ionotrope resistant shock.60\% of total babies who developes hypocalcemia was found in ionotrope resistant babies. One hypocalcemic baby developes convulsions during study period. Out of 9 babies who developes polycythemia, partial exchange transfusion done for 3 cases, which constitute $33.3 \%$ of total babies that developes polycythemia. 5 post-term were born still born, which constitute about $4.0 \%$ of the total post term deliveries. 12 post-term babies died during the study period. 8 Out 12 were due to severe birth asphyxia, which constitute $66.6 \%$ of total post-term death.

\section{Discussion}

The overall prevalence of post-term babies was $3.9 \%$. Linder $\mathrm{N}$ et $\mathrm{al}^{8}$ shows a prevalence of $3.2 \%$ which is almost correlate with our study. Advanced maternal age, primiparity and oligohydramnios are found to be the major maternal risk factor for post-term delivery. Roos N, Sahlin L, Ekman-Ordeberg G, et al ${ }^{9}$ study also shows primiparity, previous history of post-term delivery and advanced maternal age as the major risk factors for post term delivery. In addition higher BMI of mother also found to be a significant risk factors for post-term delivery which was not proved in our study and this may be due to the excistance of higher low socioeconomic status in the studied population. Roos $\mathrm{N}$ et $\mathrm{al}^{10}$ also showed advanced age as a well-established risk factor for post-term birth.

Association of post-term delivery with a higher rate of MSAF and meconium aspiration syndrome is supported by studies done Ahanya $\mathrm{SN}$ et $\mathrm{al}^{11}$.MSAF in post-term deliveries may be a sign of fetal maturity rather than a result of intrauterine insult and tendency of the baby to pass meconium once it crosses 40 weeks of gestation ${ }^{12}$.

Athar Razzaq, et $\mathrm{al}^{13}$ study shows male sex, cesarean section mode of delivery, positive pressure ventilation while resuscitation, birth asphyxia and meconium aspiration syndrome (MAS) are the major factors which is seen in 
association with PPHN. Our study shows male sex, MAS and birth asphyxia have a much higher association with PPHN. Study done by Edwin Dias and Sandeep Gada ${ }^{14}$ showed increased risk of that pre-term and post-term babies were more prone for hypoglycemia when compared to term babies and they found an incidence of $17 \%$. In our study it was found that incidence of at least one episode of hypoglycemia is $10 \%$. BRITT CLAUSSON et $\mathrm{al}^{15}$ found significant increase in the risk of stillbirth in pregnancies that continued after 41 completed gestational weeks. Same results obtained in our study too. Still birth was $2.5 \%$ in our tertiary centre but for post term pregnancies it was found to be $4.0 \%$. The increased risk of stillbirth in post-term pregnancies is partly explained by an increased rate of SGA infants. The increased risk of death among SGA infants is caused to a large extent by congenital malformations.

\section{Conclusion}

Overall, this study reported that the prevalence of post-term births to be 3.9\%.Advanced maternal age, oligohydramnios and primiparity are found to be more linked with post term delivery. Increased risk of meconium aspiration syndrome, MSAF, Birth asphyxia, polycythemia along with metabolic complications like hypocalcemia and hypoglycemia are noted during the study period. Increased risk of congenital anomalies are noted for post-term babies. This information should be keep in mind by obstetricians when counselling women regarding the management of post-term pregnancy as well as by paediatricians who need to thought of the higher rate of complications in post-term neonates.

\section{References}

1. World Health Organization. Recommended definitions, terminology and format for statistical tables related to the perinatal period and use of a new certificate for cause of perinatal deaths. Modifications recommended by FIGO as amended
October 14, 1976. Acta Obstet Gynecol Scand 1977;56:247-53.

2. Martin Ja, Hamilton be, Sutton Pd et al. births: final data for 2005. natl Vital Stat Rep. 2007; 56:1-103.

3. Kistka ZA, Palomar L, Boslaugh SE, De Baun MR, DeFranco EA, Muglia LJ. Risk for postterm delivery after previous postterm delivery. Am J Obstet Gynecol. 2007; 196: 241 e1-6.

4. Collins JW, Schulte nF, George 1 et al. Postterm delivery among African americans, Mexican americans and Whites in Chicago. ethn dis. 2001;11:181-7.

5. Clausson B, Cnattingius S, Axelsson $\mathrm{O}$. Outcomes of post-term births: the role of fetal growth restriction and malformations. Obstet Gynecol 1999;94:758-62

6. Clausson B, Cnattingius S, Axelsson O. Outcomes of post-term births: the role of fetal growth restriction and malformations. Obstet Gynecol. 1999; 94(5):758-62.

7. American College of Obstetricians and Gynecologists. Practice bulletin no. 146: management of late-term and postterm pregnancies. Obstet Gynecol 2014;124(2 Pt 1):390-6.

8. Linder N, Hiersch L, Fridman E, Klinger G, Lubin D, Kouadio F, Melamed N. Post-term pregnancy is an independent risk factor for neonatal morbidity even in low-risk singleton pregnancies. Archives of Disease in Childhood-Fetal and Neonatal Edition. 2017 Jul 1;102(4):F286-90.

9. Roos N, Sahlin L, Ekman-Ordeberg G, et al; Maternal risk factors for postterm pregnancy and cesarean delivery following labor induction. Acta Obstet Gynecol Scand. 2010 Aug89(8):1003-10.

10. Roos N, Sahlin L, Ekman-Ordeberg G, Kieler H. Maternal risk factors forpost-term pregnancy and caesarean delivery following labor induction. Acta Obstetriciaet Gynecologica. $\quad 2010 ; 89$ : 1003-10. doi:10.3109/00016349.2010.50009. 
11. Ahanya SN, Lakshmanan J, Morgan BL, et al. Meconium passage in utero: mechanisms, consequences, and management. Obstet Gynecol Surv 2005;60: 45-56.

12. David AN, Njokanma OF, Iroha E. Incidence of and factors associated with meconium staining of the amniotic fluid in a Nigerian University Teaching Hospital. J Obstet Gynaecol 2006;26:518-20.

13. Razzaq A, Quddusi AI, Nizami N. Risk factors and mortality among newborns with persistent pulmonary hypertension. Pakistan journal of medical sciences. 2013 Sep;29 (5):1099.

14. Dias E, Gada S. Glucose levels in newborns with special reference to hypoglycemia: a study from rural India. Journal of clinical neonatology. 2014 Jan;3(1):35.

15. Clausson B, Cnattingius S, Axelsson $\mathrm{O}$. Outcomes of post-term births: the role of fetal growth restriction and malformations. Obstetrics \& Gynecology. 1999 Nov 1;94(5):758-62. 